\title{
Percepção de enfermeiros sobre o cuidado nutricional à criança na Estratégia Saúde da Família
}

\author{
Nurses' perception on child nutritional care in the Family Health \\ Strategy
}

Dixis Figueroa Pedraza'

DOI: $10.1590 / 0103-1104202012410$

\begin{abstract}
RESUMO Objetivou-se analisar a percepção de enfermeiros sobre o cuidado nutricional de crianças menores de cinco anos. Trata-se de um estudo qualitativo envolvendo 18 profissionais de equipes de saúde da família de dois municípios da região metropolitana de João Pessoa, Paraíba. Realizou-se entrevista com foco na importância do desenvolvimento das ações de alimentação e nutrição para o cuidado nutricional da criança. As entrevistas foram conduzidas por meio de um roteiro de perguntas. O material foi submetido à análise de conteúdo e dela emergiram quatro categorias temáticas. Os enfermeiros apontaram a utilidade dos manuais do Ministério da Saúde para as ações de atenção nutricional e a educação em alimentação saudável como a prática mais importante desse cuidado. Entretanto, destacaram a situação socioeconômica das famílias e a capacitação dos profissionais como empecilhos para o desenvolvimento das ações. Ainda, apontaram a importância da atuação do nutricionista junto à equipe multidisciplinar para o desenvolvimento das ações de alimentação e nutrição. Além da necessidade implícita de capacitação em nutrição, evidencia-se a valorização dos documentos técnicos do Ministério da Saúde e do nutricionista para melhoria do cuidado nutricional de crianças menores de cinco anos.
\end{abstract}

PALAVRAS-CHAVE Atenção Primária à Saúde. Estratégia Saúde da Família. Serviços de saúde. Programas e políticas de nutrição e alimentação.

\begin{abstract}
The aim of this article was to analyze the perception of nurses about the nutritional care of children under five years of age. It is a qualitative study involving 18 professionals from family health teams of two municipalities in the metropolitan region of João Pessoa, Paraíba. An interview was developed focusing on the importance of developing food and nutrition actions for the nutritional care of child. The interviews were conducted through a questionnaire. The material was submitted to the content analysis and from it emerged four thematic categories. The nurses pointed out the usefulness of the manuals of the Ministry of Health for the actions of nutritional care and education in healthy food as the most important practice of this care. However, they highlight the socioeconomic situation of families and the qualification of professionals as obstacles for the development of actions. Furthermore, they also pointed out the importance of the nutritionist's work with the multidisciplinary team for the development of food and nutrition actions. In addition to the implicit need for training in nutrition, it is evident that the technical documents of the Ministry of Health and nutritionists are valued for improving the nutritional care of children under five years of age.
\end{abstract}

1 Universidade Estadual da Paraíba (UEP) - Campina Grande (PB), Brasil.

dixisfigueroa@gmail.com
KEYWORDS Primary Health Care. Family Health Strategy. Health services. Nutrition programs and policies. 


\section{Introdução}

A Estratégia Saúde da Família (ESF) constitui o modelo que sustenta a organização da Atenção Primária à Saúde (APS) no Brasil, com resultados positivos na oferta de cuidados primários de saúde e na saúde da população ${ }^{\mathbf{1 , 2}}$. Contudo, também apresenta importantes desafios em esferas como o financiamento, a formação/ educação/gestão de pessoal, o planejamento, a participação social, a integralidade e a intersetorialidade ${ }^{2}$. Essas circunstâncias convivem com altas prevalências de internações por condições sensíveis aos cuidados primários de saúde, que constituem as principais causas de hospitalizações pediátricas no Brasili3 , e com uma nova problemática de saúde caracterizada pela dupla carga da má nutrição como consequência de um processo de transição nutricional, que implica desafios adicionais e de atualização nos cuidados na área de alimentação e nutrição $0^{4}$. Ainda, a baixa orientação da APS, segundo seus atributos essenciais e as desigualdades nos serviços oferecidos, inclusive para a população infantil, indica a necessidade de mudanças estruturais e processuais condizentes com melhorias na prestação de cuidados primários de saúde e com a importância da atenção básica como coordenadora desses cuidados ${ }^{5}$.

O trabalho em equipe multiprofissional constitui um dos pilares da ESF para o alcance do novo modelo de assistência à saúde proposto. Na perspectiva da integralidade, essa conformação deve promover práticas interdisciplinares com ações de saúde desenvolvidas de forma colaborativa e mediadas pela articulação de saberes ${ }^{6}$. Nesse sentido, cabem ao enfermeiro responsabilidades relacionadas com a organização do processo de trabalho e atuar de maneira eficaz no cuidado à criança como membro da equipe mínima de saúde ${ }^{7}$. A criação dos Núcleos de Apoio à Saúde da Família (Nasf) constituiu um passo importante nessa conjuntura, com a possibilidade de inserção de profissionais da saúde e de outras áreas correlatas por meio de apoio matricial.
O número de nutricionistas na APS tem aumentado consideravelmente por meio da sua inclusão nos Nasf, sendo o terceiro profissional mais presente após o psicólogo e o fisioterapeuta. Espera-se que a atuação desse profissional possa representar ganhos à atenção básica, uma vez que as práticas nutricionais constituem prioridades dentro dos princípios de promoção da saúde e da integralidade do cuidado ${ }^{8}$.

Entretanto, sugerem-se várias barreiras relacionadas à implementação do cuidado nutricional na ESF. Destacam-se problemas na formação dos profissionais de saúde para atuarem nessa temática; fragilidade da institucionalidade da área, sobretudo nos níveis estadual e municipal; negligenciamento das ações; predomínio do modelo biomédico, curativista e hospitalocêntrico no modo de pensar e de atuar dos profissionais de saúde, inclusive do nutricionista; e abordagem inadequada na integração dos enfoques biológico e social como forma de compreender o estado nutricional ou desvalorização da importância da articulação intersetorial para a área ${ }^{9}$.

Como compo-nente central na produção do cuidado em saúde, a atenção nutricional está ancorada no tripé vigilância alimentar e nutricional, pro-moção da alimentação adequada e saudável e prevenção e controle de agravos nutricionais, devendo formar parte de ações integrais que permitam superar a fragmentação da atenção no interior das unidades básicas de saúde 4 . Segundo a Política Nacional de Atenção Integral à Saúde da Criança (PNAISC), a saúde da criança deve ser prioridade e garantida por meio de eixos estratégicos com fortes componentes nutricionais: aleitamento materno e alimentação complementar saudável, promoção e acompanhamento do crescimento e desenvolvimento integral, atenção a crianças com agravos prevalentes na infância e com doenças crônicas, atenção à saúde de crianças com necessidades especiais e de vulnerabilidade. Ainda, destaca a importância de fortalecer a participação e o controle social no planejamento, execução, monitoramento e avaliação das intervenções de atenção integral à saúde da criança ${ }^{10}$. Nesse 
sentido, destaca-se que pesquisas sobre a área de alimentação e nutrição na APS ainda são escassas, sobretudo as com foco na avaliação das ações e programas ${ }^{11}$.

O objetivo deste estudo foi analisar a percepção de enfermeiros sobre o cuidado nutricional de crianças menores de cinco anos.

\section{Material e métodos}

Estudo qualitativo descritivo, envolvendo equipes de saúde da ESF de dois municípios do estado da Paraíba. A escolha dos municípios considerou suas semelhanças com relação à posição geográfica (localização na região metropolitana de João Pessoa, capital do estado da Paraíba, com acesso à rede de serviços nela disponibilizados), ao grau de urbanização (de quase 100\%), ao desenvolvimento (índice de desenvolvimento humano de nível médio - 0,649 em um dos municípios, e 0,748 no outro) e à tradição na organização de serviços primários de saúde (cobertura da ESF de $100 \%)$. Um dos municípios tem população de 99.716 habitantes, dos quais, 7.862 são crianças menores de cinco anos, e possui sistema de saúde composto por 28 equipes da ESF. O outro município tem população de 57.944 habitantes, dos quais, 4.596 são crianças menores de cinco anos, e possui sistema de saúde composto por 19 equipes da ESF.

Em cada município, foram sorteadas aleatoriamente nove equipes de saúde para participar da pesquisa. Em cada equipe de saúde, foi realizada uma entrevista com o enfermeiro.

Os dados foram coletados entre os meses de agosto e dezembro de 2014 nas unidades de saúde. Inicialmente, os enfermeiros foram caracterizados segundo sexo, idade, tempo de vínculo na equipe de saúde e participação em cursos de capacitação para atuar na APS.

Realizou-se entrevista com os enfermeiros, com foco na importância do desenvolvimento das ações de alimentação e nutrição direcionadas para o cuidado nutricional das crianças menores de cinco anos na ESF, seguindo um roteiro de perguntas com seis questões norteadoras: i. aspectos mais importantes para trabalhar as demandas de alimentação e nutrição, ii. conhecimentos importantes para trabalhar as demandas de alimentação e nutrição, iii. obstáculos para trabalhar as demandas de alimentação e nutrição, iv. fontes de informação usadas para transmitir orientações sobre alimentação e nutrição, v. importância da implementação de rotinas ou protocolos de cuidado nutricional, e vi. principais práticas de alimentação e nutrição desenvolvidas na rotina de trabalho. As entrevistas tiveram tempo médio de duração de 30 minutos, foram gravadas em Gravador Digital de Áudio Voz Sony Icd-px240 e transcritas literalmente (ipsis verbis).

Fez-se análise de conteúdo temática do material empírico ${ }^{12}$ mediante: i. leitura exaustiva das entrevistas e identificação dos núcleos de sentido, ii. agrupamento dos temas afins, e iii. construção das categorias empíricas. O processo foi orientado pelo objeto, pelos objetivos e fundamentos do estudo. Dessa análise, emergiram quatro categorias temáticas relacionadas ao desenvolvimento das ações de alimentação e nutrição em termos de facilidades, obstáculos, principais práticas e necessidades.

A pesquisa foi aprovada pelo Comitê de Ética em Pesquisa da Universidade Estadual da Paraíba, com o protocolo de número 19689613.3.0000.5187. Todos os participantes assinaram o Termo de Consentimento Livre e Esclarecido. Para resguardar a identidade dos entrevistados, os profissionais foram identificados pelas denominações ‘E1, E2, E3...’.

\section{Resultados e discussão}

Todos os entrevistados eram mulheres e tinham mais de 30 anos, o que pode ser justificado pelo forte predomínio de profissionais de enfermagem do sexo feminino e na faixa etária de adulto jovem, o que relaciona-se a um processo histórico e reproduz a força de trabalho da APS no País ${ }^{13-15}$. Apenas sete profissionais possuíam vínculo estável mínimo 
de dois anos, característica que pode estar relacionada à precarização do trabalho em saúde, dadas as dificuldades de realização de concurso público e de boas remunerações, que contribuem para a rotatividade profissional, com possíveis prejuízos nos serviços prestados à população ${ }^{15-17}$. Dos 18 participantes do estudo, 13 tinham frequentado algum curso de capacitação para atuar na APS, resultado que assemelha-se à situação encontrada entre enfermeiros de Goiânia ${ }^{18}$ e pode derivar da localização dos municípios estudados, tendo em vista as melhores oportunidades de cursos nos grandes centros urbanos do País ${ }^{19}$. No contexto do presente trabalho, é possível presumir enriquecimento dos conteúdos das falas associado à capacitação dos profissionais.

As falas e o agrupamento de ideias, analisados de acordo com o objetivo do estudo, levaram à formação de quatro categorias centrais que representam o contexto da compreensão, percepção e atuação dos enfermeiros nas ações de alimentação e nutrição. As categorias centrais elucidativas dos aspectos do trabalho valorizados por enfermeiros no tocante às ações de alimentação e nutrição foram: 1 . facilidades para o desenvolvimento das ações de alimentação e nutrição: manuais do Ministério da Saúde, 2. obstáculos para o desenvolvimento das ações de alimentação e nutrição: situação socioeconômica das famílias, 3. práticas de maior importância na área de alimentação e nutrição: educação sobre alimentação saudável, e 4. necessidades para o desenvolvimento das ações de alimentação e nutrição: capacitação profissional e trabalho multidisciplinar com a atuação de nutricionista.

\section{Facilidades para o desenvolvimento das ações de alimentação e nutrição: manuais do Ministério da Saúde}

Na primeira categoria, os profissionais reforçaram a relevância dos manuais do Ministério da Saúde como elementos facilitadores para o desenvolvimento das ações de alimentação e nutrição.
A gente tem guias do Ministério da Saúde. De vez em quando, a secretaria de saúde manda folders, mas também não são todas as vezes. A gente pesquisa muito na internet, a gente monta os formulariozinhos para eles responderem, a gente traz cartazes. (E1).

Usamos sempre os manuais do Ministério. A gente recebe algumas portarias... A gente recebe portaria, eles mandam portarias, mas a gente nunca foi treinado pra isso. (E2).

Eu me baseio nos manuais do Ministério que a gente tem, nas informações da internet, né? (E3).

Dispor de material técnico respalda as ações realizadas pelos enfermeiros e representa um dos principais fatores para que se tenha sucesso nas consultas de enfermagem ${ }^{7,20}$. A adequada utilização dessas tecnologias torna possível a padronização e a sistematização dos cuidados, permitindo sua qualificação ${ }^{21,22}$. Para o desenvolvimento das ações de alimentação e nutrição, vários documentos técnicos do Ministério da Saúde estão disponíveis, sendo sua utilização importante para o diagnóstico correto do estado nutricional, a identificação de riscos nutricionais e a implantação de ações e programas prioritários 21,23,24. Entretanto, estudos têm relatado a disponibilidade insuficiente desses materiais nas unidades de saúde e dificuldades no seu uso $\mathbf{2 1 , 2 3 , 2 5}$, conforme relatado pelos enfermeiros do presente estudo, o que pode advir das suas limitações nas recomendações relacionadas às soluções exigidas do profissional de saúde na sua prática diária ${ }^{26}$. Destarte, reforça-se a importância da capacitação para uma melhor apropriação desses instrumentos ${ }^{\mathbf{2 1}}$.

Adicionalmente, conforme estudo internacional que ressaltou que enfermeiros recorrem aos recursos informacionais de mais fácil acesso e de respostas rápidas, como informações disponíveis na internet ${ }^{27}$, os profissionais do atual trabalho também manifestaram o uso de material obtido por essa via. Vale questionar que tipo de documento 
na internet os enfermeiros acessaram, como forma de ter garantia da sua qualidade. Como isso não foi possível de ser constatado neste estudo, recomenda-se o planejamento de pesquisas adicionais para esses fins. Cabe ressaltar que a valorização dos manuais do Ministério da Saúde mostrada pelos profissionais que participaram desta pesquisa revela a importância de estarem disponíveis para uso nas unidades de saúde.

\section{Obstáculos para o desenvolvimento das ações de alimentação e nutrição: situação socioeconômica das famílias}

Na segunda categoria, a situação socioeconômica das famílias foi abordada como importante limitador relacionado à capacidade de se ter uma boa alimentação e no desenvolvimento das ações de alimentação e nutrição.

O pessoal não tem muita condição social de realmente se manter com uma boa alimentação [...] não tem muito dinheiro pra verdura, não tem condições de comprar uma fruta, por exemplo, três vezes por semana. O pessoal não se alimenta direito, e é a grande maioria. (E2).

A baixa renda do pessoal, logo a gente trabalha com uma população muito carente [...] vem uma paciente aqui e aí a gente quer, uma criança, orientar, aí a gente manda pra nutricionista. Chegar lá, ela vai passar uma dieta. Nem sempre, na maioria das vezes, ela não tem condição de seguir aquela dieta [...] eles não querem nem ouvir, por isso que eles nem vêm pro posto. (E4).

Como é que você vai falar em nutrição, como é que você vai falar em alimentação se as pessoas não têm condição de comprar até o próprio alimento, né? [...] quando a gente orienta o paciente a ir falar com o nutricionista, ele diz logo: 'eu não vou não, que eu não tenho dinheiro pra comprar aqueles negócios que elas passa'. (E7).

A fragilidade socioeconômica de famílias como as do presente estudo é tida como um dos principais responsáveis pela insegurança alimentar e nutricional. A relação entre esses dois fatores determina modificações na qualidade da alimentação, condicionadas ao baixo poder aquisitivo, priorizando-se o consumo de alimentos de alta densidade energética, que contribuem para a saciedade. As baixas condições socioeconômicas implicam a diminuição da quantidade de alimento que pode ser acessado, com impacto direto no bem-estar e na qualidade de vida ${ }^{28}$. Assim como o presente estudo, outros também relataram as condições socioeconômicas das famílias atendidas na ESF como obstáculo para o desenvolvimento das ações pelos profissionais de saúde, reforçando-se a importância da compreensão do modelo de produção social da saúde e do estado nutricional ${ }^{\mathbf{2 3}, 29}$. Assim, a formação dos profissionais, além do foco nos determinantes sociais da saúde, deve agregar bases relacionadas ao perfil epidemiológico e necessidades da população e de grupos vulneráveis, com vivências teóricas e práticas voltadas para a realidade local ${ }^{30}$.

\section{Práticas de maior importância na área de alimentação e nutrição: educação sobre alimentação saudável}

$\mathrm{Na}$ terceira categoria, os profissionais deixaram clara a importância da educação com abordagem direcionada à alimentação saudável para que as mães das crianças ampliem seus conhecimentos sobre o tema. Entretanto, percebe-se tanto nas falas quanto na carência de argumentos que não há uma construção adequada do processo para a educação alimentar e nutricional.

Tentar sensibilizar essas mães, para, assim, pra ter uma alimentação saudável. Já pra evitar, né? Problemas futuros como, no caso a obesidade, que a gente vê que ultimamente tem aumentado bastante devido a esse erro alimentar. (E5).

Eles têm que entender que o que a gente tenta passar pra eles sobre alimentação é o melhor, 
né? Acho que a gente só consegue isso através de conversa, conversa até mesmo amigáveis. Não adianta você querer impor nada às mães [...] tem que ser um trabalho de formiguinha mesmo. (E9).

É competência dos enfermeiros que fazem parte das equipes da ESF a educação em saúde, sendo responsáveis por consultas de enfermagem e atividades em grupo ${ }^{31}$. Os enfermeiros reconhecem por meio dessa categoria a importância de atividades educativas sobre alimentação saudável, o que pode ser reflexo da educação em saúde como prática relevante desse profissional ${ }^{32,33}$. Além disso, o enfermeiro tem se preocupado com a busca de fundamentação teórica e prática para o desempenho do cuidado nutricional da criança ${ }^{34}$.

Entretanto, estudos têm mostrado que a educação em saúde desenvolvida por enfermeiros é deficiente, associando-se a fatores como as crenças e os costumes das comunidades, a desvalorização profissional, a visão hegemônica centrada na doença na forma de pensar e as precárias condições materiais ${ }^{33,35}$. Assim sendo, a promoção da alimentação saudável requer de enfermeiros capacitação para tal, uma vez que são eles os principais envolvidos nas ações de nutrição nas unidades de saúde, sem que contem, na maioria das vezes, com os conhecimentos necessários para esses fins ${ }^{23,29}$. Além disso, para um cuidado nutricional efetivo, o serviço de saúde deve estar organizado de forma a permitir um processo constante de formação, ressaltando-se que a capacitação nem sempre resulta em educação permanente. Nesse sentido, por meio de um estudo desenvolvido em 28 municípios brasileiros de grande porte, sugeriu-se que a educação permanente em alimentação e nutrição viabiliza-se com a disponibilidade de recursos e parcerias, enquanto tem como fatores dificultadores a indisponibilidade de agendas e a falta de profissionais na gestão das ações de nutrição. Adicionalmente, destacam-se as ações educativas a partir do nível local, considerando as necessidades dos profissionais e da comunidade ${ }^{36}$.

\section{Necessidades para o desenvolvimento das ações de alimentação e nutrição: capacitação profissional e trabalho multidisciplinar com a atuação de nutricionista}

A quarta categoria trata a necessidade que os profissionais sentem de capacitação para atuar na área de nutrição, além da importância da integração da equipe de saúde mediante a multidisciplinaridade e a participação do nutricionista.

Acho que primeiro a gente tinha que saber classificar. Nem todo profissional sabe avaliar uma criança pra saber se uma criança tá desnutrida ou não, o acompanhamento dessa criança [...] e a gente ter realmente noções de orientação alimentar, porque a gente não tem. A gente só tem quando a gente vai em busca. (E3).

O nutricionista ele é, é como eu te falei, ele é de grande importância na unidade, mas eu acharia que todos os profissionais tivessem pelo menos um pouquinho do nutricionista, né? Que a gente tivesse um pouquinho do entendimento também. (E4).

Acho que a gente precisa saber mais sobre alimentos, as propriedades dos alimentos, essas coisas. A importância de cada fase, de cada nutriente, de cada coisa que a criança precisa mais, entendeu? E tentar fazer isso de uma maneira acessível pra eles, uma comida assim que ele tenha capacidade de obter. (E6).

Tudo que envolva a criança, crescimento e desenvolvimento, a gente nunca teve capacitação nenhuma [...] eu acho necessário ter nem que seja um minicurso. (E7).

Percebe-se, como em estudo anterior ${ }^{29}$, o despreparo dos enfermeiros na área de nutrição, principalmente devido à falta de conhecimento técnico, o que pode comprometer a implantação das ações de alimentação e nutrição e prejudicar a integralidade do cuidado, 
alertando para a necessidade de capacitação desses profissionais $\mathbf{2 1 2}^{\mathbf{2 4}, \mathbf{2 9}}$. Apesar de a enfermagem representar uma profissão idônea para contribuir de maneira significativa para a melhoria do estado nutricional da população ${ }^{37}$, as ações de alimentação e nutrição caracterizam-se por sua interdisciplinaridade ${ }^{29}$, como levantado nas entrevistas. Essa conjuntura vai de encontro à Política Nacional de Alimentação e Nutrição, que prevê a garantia de educação permanente em alimentação e nutrição para os trabalhadores de saúde ${ }^{\mathbf{3 8}}$. Nesse sentido, é pertinente destacar a importância da educação interprofissional no processo de formação dos profissionais de saúde, com foco em práticas colaborativas que possam impactar o modelo de atenção à saúde ${ }^{39}$. Sob esse aspecto, iniciativas como o Programa de Educação pelo Trabalho para a Saúde e a implantação dos Nasf configuram-se como importantes referências alicerçadas às premissas da educação interprofissional e do trabalho em equipe de forma colaborativa ${ }^{\mathbf{4 0 , 4 1}}$.

Cabe ressaltar que as deficiências atingem até ações básicas, como a vigilância do crescimento e do desenvolvimento, recomendada como um dos eixos estratégicos do cuidado à saúde da criança como parte da PNAISC, na qual a APS representa a coordenadora e o ponto central da assistência ${ }^{10}$. Essas fragilidades também foram anteriormente sistematizadas por meio de uma revisão da literatura que alerta sobre a probabilidade de efeitos negativos na promoção da saúde infantil e na prevenção de desfechos indesejados, além de constituir importante indicador da qualidade dos serviços ${ }^{21}$.

Por meio da análise das entrevistas, foi possível constatar, também, a importância atribuída ao trabalho do nutricionista na equipe multidisciplinar, conforme achados anteriores que destacam esse profissional como detentor de conhecimentos específicos para atuar no âmbito das questões de alimentação e nutrição, de forma a conscientizar a população sobre as consequências de uma má alimentação ${ }^{\mathbf{2 3} 29,42}$. O nutricionista é o único profissional capacitado para realizar diagnósticos nutricionais e propor orientações dietéticas adequadas, podendo sua atuação contribuir com outros profissionais para o fortalecimento dos conhecimentos técnicos sobre a temática e a implantação das ações da área 9,23,24,29,42. Na APS, o nutricionista tem função de caráter predominantemente coletivo e centrado na comunidade, na perspectiva de contribuir para o planejamento e a organização do cuidado nutricional, em sintonia com a qualificação e a resolutividade dos serviços ${ }^{\mathbf{4 3}, 44}$. Dessa forma, salienta-se a relevância do nutricionista para o campo de práticas do enfermeiro com relação à alimentação e à nutrição na APS, que destaca-se, a despeito de importantes lacunas na formação e nos conhecimentos em nutrição, por ser o profissional com maior envolvimento no cuidado nutricional e por seu papel fundamental na atenção à saúde da criança, com ações para a construção de conhecimentos sobre nutrição, a promoção da alimentação saudável e o aconselhamento nutricional 24,37,42,45-47.

Como limitações deste estudo, deve-se considerar que se restringiu a dois municípios de um estado da região Nordeste do Brasil, analisando, exclusivamente, a perspectiva dos enfermeiros, os quais podem apresentar uma visão dos serviços oferecidos diferente da de outras categorias profissionais e de outros atores sociais, como usuários e gestores. Além disso, as características intrínsecas à abordagem metodológica qualitativa, na qual a representatividade dos sujeitos é desconsiderada, não possibilitam a generalização dos resultados.

\section{Conclusões}

Os achados deste estudo propiciaram conhecer a percepção de enfermeiros sobre a importância do cuidado nutricional de crianças menores de cinco anos. Os resultados encontrados poderão servir de alerta para todos os profissionais de saúde que atuam diretamente 
*Orcid (Open Researcher and Contributor ID). na atenção à criança, pois os discursos apresentados, além de ajudarem na compreensão sobre o cuidado nutricional da criança, mostram a necessidade de maior divulgação e sensibilização com os problemas nos serviços de atenção básica à saúde.

Os discursos apresentados pelos enfermeiros evidenciaram que o desenvolvimento das ações de alimentação e nutrição na APS confronta dificuldades frente às barreiras do próprio sistema de saúde relacionadas à qualificação para trabalhar essa temática, bem como às demandas impostas pela situação socioeconômica da clientela. As práticas educativas sobre alimentação saudável foram as mais valorizadas. Considerando a importância atribuída pelos profissionais, as ações podem ser facilitadas com o uso dos manuais do Ministério da Saúde existentes para esses fins e com a atuação do nutricionista junto à equipe multidisciplinar, além da necessidade implícita de capacitação em nutrição.

Dessa forma, percebe-se um desafio na formação de trabalhadores para atuarem no SUS, com foco na educação permanente. Essa premissa, acompanhada de outras mudanças, a exemplo da garantia de estruturas mínimas para as ações de alimentação e nutrição, da valorização profissional e da participação social como forma de interferir nos interesses coletivos relacionados às políticas de saúde, expressam circunstâncias da gestão democrática e do modelo de cuidado centrado no usuário, indispensáveis à satisfação com a atenção à saúde da criança na ESF.

\section{Colaborador}

Figueroa Pedraza D (0000-0002-5394-828X)* é responsável pela elaboração do manuscrito.

\section{Referências}

1. Harris M, Haines A. Brazil's Family Health Programme. Br M J. 2010 [acesso em 2018 jan 10]; 341:c4945. Disponível em: https://www.researchgate.net/publication/232266318_Brazil's_Family_Health_Programme.

2. Arantes LJ, Shimizu HE, Merchán-Hamann E. Contribuições e desafios da Estratégia Saúde da Família na Atenção Primária à Saúde no Brasil: revisão da literatura. Ciênc. Saúde Colet. 2016; 21(5):1499-1509.
3. Prezotto KL, Chaves MMN, Mathias TAF. Hospitalizações sensíveis à atenção primária em crianças, segundo grupos etários e regionais de saúde. Rev. Esc. Enferm. USP. 2015; 49(1):44-53.

4. Jaime PC, Delmuè DCC, Campello T, et al. Um olhar sobre a agenda de alimentação e nutrição nos trinta anos do Sistema Único de Saúde. Ciênc. Saúde Colet. 2018; 23(6):1829-36. 
5. Damasceno SS, Nóbrega VM, Coutinho SED, et al. Saúde da criança no Brasil: orientação da rede básica à Atenção Primária à Saúde. Ciênc. Saúde Colet. 2016; 21(9):2961-73.

6. Jacowski M, Budal AMB, Lemos DS, et al. Trabalho em equipe: percepção dos profissionais da Estratégia de Saúde da Família. Rev. Baiana Enf. 2016; 30(2):1-9.

7. Souza RS, Ferrari RAP, Santos TFM, et al. Atenção à saúde da criança: prática de enfermeiros da saúde da família. Rev. Min. Enferm. 2013; 17(2):331-9.

8. Vasconcelos IAL, Sousa MF, Santos LMP. Evolução do quantitativo de nutricionistas na Atenção Básica do Brasil: a contribuição dos Núcleos de Apoio à Saúde da Família e da Estratégia Saúde da Família de 2007 a 2013. Rev. Nutr. 2015; 28(4):431-50.

9. Rigon SA, Schmidt ST, Bógus CM. Desafios da nutrição no Sistema Único de Saúde para construção da interface entre a saúde e a segurança alimentar e nutricional. Cad. Saúde Pública. 2016 [acesso em 2018 jan 10]; 32(3):e00164514. Disponível em: http://www. scielo.br/scielo.php?pid=S0102311X2016000300709 $\&$ script=sci_abstract\&tlng=pt.

10. Brasil. Ministério da Saúde. Portaria GM nº 1130, de 5 de agosto de 2015. Institui a Política Nacional de Atenção Integral à Saúde da Criança (PNAISC) no âmbito do Sistema Único de Saúde (SUS). Diário Oficial da União. 5 Ago 2015. [acesso em 2020 jan 20]. Disponível em: http://bvsms.saude.gov.br/bvs/saudelegis/gm/2015/prt1130_05_08_2015.html.

11. Nunes Pereira T, Monteiro RA, Santos LMP. Alimentación y nutrición en atención primaria en Brasil. Gac. Sanit. 2018; 32(3):297-303.

12. Minayo MCS. O desafio do conhecimento: pesquisa qualitativa em saúde. 9. ed. São Paulo: Hucitec; 2006.

13. Thumé E, Fehn AC, Acioli S, et al. Formação e prática de enfermeiros para a Atenção Primária à Saúde - avanços, desafios e estratégias para fortalecimento do Sistema Único de Saúde. Saúde debate. 2018; 42(espl):275-88.
14. Tambasco LP. A satisfação no trabalho da equipe multiprofissional que atua na Atenção Primária à Saúde. Saúde debate. 2017; 41(esp2):140-51.

15. Oliveira MM, Figueroa Pedraza D. Contexto de trabalho e satisfação profissional de enfermeiros que atuam na Estratégia Saúde da Família. Saúde debate. 2019; 43(122):763-77.

16. Alvarenga EC, Oliveira PTR, Pinheiro HHC, et al. Condições de trabalho de Equipes de Saúde da Família do Pará. Rev. NUFEN. 2018; 10(1):58-72.

17. Rolim MD, Lima SML, Barros DC, et al. Avaliação do SISVAN na gestão de ações de alimentação e nutrição em Minas Gerais, Brasil. Ciênc. Saúde Colet. 2015; 20(8):2359-69.

18. Oliveira MPR, Menezes IHCF, Sousa LM, et al. Formação e qualificação de profissionais de saúde: fatores associados à qualidade da Atenção Primária. Rev. Bras. Educ. Med. 2016; 40(4):547-59.

19. Mattos LB, Dahmer A, Magalhães CR. Contribuições do curso de especialização em Atenção Primária à Saúde à prática de profissionais da saúde. ABCS Health Sci. 2015; 40(3):184-189.

20. Kahl C, Meirelles BHS, Lanzoni GMM, et al. Actions and interactions in clinical nursing practice in Primary Health Care. Rev. Esc. Enferm. USP. 2018 [acesso em 2018 fev 14]; 52:e03327. Disponível em: http://www.scielo.br/scielo.php?script=sci_ab stract\&pid=S008062342018000100415\&lng=en\& $\mathrm{nrm}=$ iso.

21. Figueroa Pedraza D. Growth surveillance in the context of the Primary Public Healthcare Service Network in Brazil: literature review. Rev. Bras. Saúde Matern. Infant. 2016; 16(1):7-19.

22. Figueroa Pedraza D, Santos IS. Profile and performance of nutritionists in Primary Health Care. Rev. Nutri. 2017; 30(6):835-45.

23. Pedraza DF, Menezes TN, Costa GMC. Ações de alimentação e nutrição na estratégia saúde da fa- 
mília: estrutura e processo de trabalho. Rev. Enferm. UERJ. 2016; 24(4):e15848.

24. Junqueira TS, Cotta RMM. Matriz de ações de alimentação e nutrição na Atenção Básica de Saúde: referencial para a formação do nutricionista no contexto da educação por competências. Ciênc. Saúde Colet. 2014; 19(5):1459-74.

25. Venancio SI, Giugliani ERJ, Silva OLO, et al. Associação entre o grau de implantação da Rede Amamenta Brasil e indicadores de amamentação. Cad. Saúde Pública. 2016 [acesso em 2018 fev 14]; 32(3):e00010315. Disponível em: http://www.scielo.br/scielo.php?pid=S0102311X2016000300704\&s cript=sci_abstract\&tlng=pt.

26. Baralhas M, Pereira MAO. Prática diária dos agentes comunitários de saúde: dificuldades e limitações da assistência. Rev. Bras. Enferm. 2013; 66(3):358-65.

27. Thorsteinsson HS. Icelandic nurses' beliefs, skills, and resources associated with evidence-based practice and related factors: a national survey. Worldviews Evid Based Nurs. 2013; 10(2):116-26.

28. Teo CRPA, Ferraz L, Cembranel F. Agentes comunitários de saúde: percepções sobre alimentação no contexto da Estratégia Saúde da Família. Ciênc. Cuid. Saúde. 2015; 14(3):1229-36.

29. Pimentel VRM, Sousa MF, Hamann EM, et al. Alimentação e nutrição na Estratégia Saúde da Família em cinco municípios brasileiros. Ciênc. Saúde Colet. 2014; 19(1):49-57.

30. Machado MH, Ximenes Neto FRG. Gestão da Educação e do Trabalho em Saúde no SUS: trinta anos de avanços e desafios. Ciênc. Saúde Colet. 2018; 23(6):1971-80.

31. Brasil. Ministério da Saúde. Portaria n ${ }^{\circ} 2.436$, de 21 de setembro de 2017. Aprova a Política Nacional de Atenção Básica, estabelecendo a revisão de diretrizes para a organização da Atenção Básica, no âmbito do Sistema Único de Saúde (SUS) [internet]. Diá- rio Oficial da União. 31 Set 2017. [acesso em 2018 nov 11]. Disponível em: http://www.brasilsus.com.br/index.php/legislacoes/gabinete -do- -ministro/16247-portaria-n-2-436-de-21-de-setembro-de-2017.

32. Barbiani R, Dalla Nora CR, Schaefer R. Nursing practices in the primary health care context: a scoping review. Rev Latino-Am Enfermagem. 2016 [acesso em fev 2018 14]; 24:e2721. Disponível em: http:// www.scielo.br/scielo.php?script=sci_arttext\&pid =S0104-11692016000100609.

33. Moreno CA, Ferraz LR, Rodrigues TS, et al. Atribuições dos profissionais de enfermagem na Estratégia de Saúde da Família, uma revisão das normas e práticas. Rev. Bras. Ciên. Saúde. 2015; 19(3):233-40.

34. Monteiro FPM, Caetano JA, Araújo TL. Enfermagem na saúde da criança: estudo bibliográfico acerca da avaliação nutricional. Rev. Enferm. Anna Nery. 2010; 14(2):406-11.

35. Moutinho CB, Almeida ER, Leite MTS, et al. Dificuldades, desafios e superações sobre educação em saúde na visão de enfermeiros de saúde da família. Trab. Educ. Saúde. 2014; 12(2):253-72.

36. Ricardi LM, Sousa MF. Educação permanente em alimentação e nutrição na Estratégia Saúde da Família: encontros e desencontros em municípios brasileiros de grande porte. Ciênc. Saúde Colet. 2015; 20(1):20918.

37. Hidalgo MCM, López LP. Intervenciones enfermeras aplicadas a la nutrición. Nutr. Clín. Diet. Hosp. 2017; 37(4):189-93.

38. Brasil. Ministério da Saúde. Política Nacional de Alimentação e Nutrição. Brasília, DF: Ministério da Saúde; 2013.

39. Freire Filho JR, Silva CBG, Costa MV, et al. Educação Interprofissional nas políticas de reorientação da formação profissional em saúde no Brasil. Saúde debate. 2019; 43(esp1):86-96. 
40. Magnago C, França T, Belisário SA, et al. PET-Saúde/ GraduaSUS na visão de atores do serviço e do ensino: contribuições, limites e sugestões. Saúde debate. 2019; 43(esp1):24-39.

41. Bispo Júnior JP, Moreira DC. Cuidado colaborativo entre os núcleos de apoio à saúde da família e as equipes apoiadas. Physis. 2018 [acesso em 2018 fev 14]; 28(3):e280310. Disponível em: http:// www.scielo.br/scielo.php?script=sci_arttext\&pid =S010373312018000300605.

42. Fittipaldi ALM, Barros DC, Romano VF. Apoio Matricial nas ações de Alimentação e Nutrição: visão dos profissionais. Physis. 2017; 3:793-811.

43. Conselho Federal de Nutricionistas. O papel do nutricionista na atenção primária à saúde. 3. ed. Brasília, DF: Conselho Federal de Nutricionistas; 2015.

44. Brasil. Ministério da Saúde. Matriz de ações de alimentação e nutrição na Atenção Básica à Saúde. Brasília, DF: Ministério da Saúde; 2009. (Séria A. Normas e Manuais Técnicos).
45. Palombo CNT, Fujimori E, Toriyama ATM, et al. Difficulties in nutritional counseling and child growth follow-up: from a professional perspective. Rev. Bras. Enferm. 2017; 70(5):949-57.

46. Campos AAO, Cotta RMM, Oliveira JM, et al. Aconselhamento nutricional de crianças menores de dois anos de idade: potencialidades e obstáculos como desafios estratégicos. Ciênc. Saúde Colet. 2014; 19(2):529-38.

47. Einloft ABN, Cotta RMM, Araújo RMA. Promoção da alimentação saudável na infância: fragilidades no contexto da ação básica. Ciênc. Saúde Colet. 2018;23(1):6172

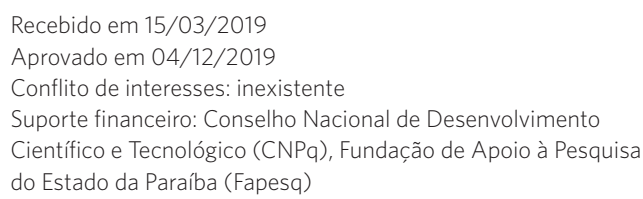

